\title{
On Basis Properties of Degenerate Exponential System
}

\author{
Mamedova Zahira \\ Non-Harmonic Analysis, Institute of Mathematics and Mechanics of NAS of Azerbaijan, Baku, Azerbaijan \\ Email: zahira_eng13@hotmail.com
}

Received September 5, 2012; revised October 10, 2012; accepted October 17, 2012

\begin{abstract}
Exponential systems of the form $\left\{\mu(x) \mathrm{e}^{i n x}\right\}_{n \in Z_{0}}$ are considered, where $\mu(x)$ is a degenerate coefficient, $\mathbf{Z}$ is a set of all integers and $Z_{0} \subset \mathbf{Z}$. The basis properties of these systems in $L_{p}(-\pi, \pi), 1 \leq p<+\infty$, when, generally speaking, $\mu(x)$ doesn't satisfy the Muckenhoupt condition are investigated.
\end{abstract}

Keywords: System of Exponents; Degeneration; Basicity; Completeness; Minimality

\section{Introduction}

Basis properties of classical system of exponents $\left\{\mathrm{e}^{\mathrm{int}}\right\}_{n \in \mathbf{Z}}$ in Lebesgue spaces $L_{p}(-\pi, \pi), 1 \leq p<+\infty$, are well studied (see e.g. [1-4]). N. K. Bari in her fundamental work [5] raised the issue of the existence of normalized basis in $L_{2}$, which is not Riesz basis. The first example of this was given by K. I. Babenko [6]. He proved that the degenerate system of exponents $\left\{|x|^{\alpha} e^{\text {int }}\right\}_{n \in \mathbf{Z}}$ with $|\alpha|<\frac{1}{2}$ forms a basis for $L_{2}(-\pi, \pi)$, but is not Riesz basis when $\alpha \neq 0$. This result has been extended by V. F. Gaposhkin [7]. In [8] the condition on the weight $\rho$ was found, which make the system $\left\{e^{\text {int }}\right\}_{n \in \mathbf{Z}}$ form a basis for the weight space $L_{p, \rho}(-\pi, \pi)$ with a norm

$$
\|f\|_{p, \rho}=\left(\int_{-\pi}^{\pi}|f(t)|^{p} \rho(t) \mathrm{d} t\right)^{\frac{1}{p}} .
$$

Similar problems are considered in [9-13]. Basis properties of a degenerate system of exponents are closely related to the similar properties of an ordinary system of exponents in corresponding weight space. In all the mentioned works the authors consider the cases, when the weight or the degenerate coefficient satisfies the Muckenhoupt condition (see, for example, [14]).

In this paper the basis properties of exponential systems with a degenerate coefficient are studied in the spaces $L_{p}(-\pi, \pi), 1 \leq p<+\infty$, when the degenerate coefficient does not satisfy the Muckenhoupt condition. A similar problem was considered earlier in [15].

\section{Completeness and Minimality}

We consider a system of exponents

$$
\left\{E_{n}(\mu)\right\}_{n \in \mathbf{Z}} \equiv\left\{\mu(t) \mathrm{e}^{\mathrm{int}}\right\}_{n \in \mathbf{Z}},
$$

with a degenerate coefficient

$$
\mu(t)=\prod_{k=0}^{r}\left|t-t_{k}\right|^{\alpha_{k}},
$$

where $t_{0}=0,0 \neq t_{k} \in(-\pi, \pi], k=\overline{1, r}$, are different points. It is clear that $\left\{E_{n}\right\}_{n \in \mathbf{Z}} \subset L_{p} \equiv L_{p}(-\pi, \pi), 1 \leq p<+\infty$, if and only if $\alpha_{k} p>-1, k=\overline{1, r}$. Assume that the function $f \in L_{q}\left(\frac{1}{p}+\frac{1}{q}=1\right)$ cancels the system $\left\{E_{n}\right\}_{n \in \mathbf{Z}}$ out, that is

$$
\int_{-\pi}^{\pi} \mu(t) \mathrm{e}^{\mathrm{int}} \bar{f}(t) \mathrm{d} t=0, \forall n \in \mathbf{Z}
$$

where $(-)$ is a complex conjugate. It is clear that $F \in L_{1}(-\pi, \pi)$, where $F=\mu f$. Then, it follows directly from (2) that $F=0$ a.e. on $(-\pi, \pi)$ and, consequently, $f=0$ a.e. on $(-\pi, \pi)$. Thus, if

$$
\left\{\alpha_{k}\right\}_{0}^{r} \subset\left(-\frac{1}{p},+\infty\right)
$$

then system $\left\{E_{n}\right\}_{n \in \mathbf{Z}}$ is complete in

$$
L_{p}(-\pi, \pi), 1 \leq p<+\infty \text {. }
$$

Now consider the minimality of system $\left\{E_{n}\right\}_{n \in \mathbf{Z}}$ in $L_{p}(-\pi, \pi)$. If

$$
\left\{\alpha_{k}\right\}_{0}^{r} \subset\left(-\frac{1}{p}, \frac{1}{q}\right)
$$


then it is minimal in $L_{p}(-\pi, \pi)$ and system $\left\{E_{n}\left(\mu^{-1}\right)\right\}_{n \in \mathbf{Z}}$ is biorthogonal to it. So in this case $E_{\mu^{-1}} \subset L_{q}(-\pi, \pi)$. Let $\alpha_{0} \in\left[\frac{1}{q}, 1+\frac{1}{q}\right)$. Consider the system

$$
\left\{\mu^{-1}(t)\left(\mathrm{e}^{\mathrm{int}}-1\right)\right\}_{n \neq 0} \text {. }
$$

We have

$$
\begin{aligned}
& \int_{-\pi}^{\pi} \mu(t) \mathrm{e}^{\mathrm{int}} \mu^{-1}(t)\left(\mathrm{e}^{-i m t}-1\right) \mathrm{d} t \\
& =\int_{-\pi}^{\pi} \mathrm{e}^{i(n-m) t} \mathrm{~d} t \int_{-\pi}^{\pi} \mathrm{e}^{\mathrm{int}} \mathrm{d} t=2 \pi \delta_{n m}, \forall n, m \neq 0 .
\end{aligned}
$$

It is clear that in the neighborhood of zero it holds

$$
\mathrm{e}^{\mathrm{int}}-1 \sim-\frac{n^{2}}{2} t .
$$

Consequently, the following representation

$$
\mu^{-1}(t)\left(\mathrm{e}^{\mathrm{int}}-1\right) \sim-\frac{n^{2}}{2} t^{1-\alpha_{0}} \prod_{k=1}^{r}\left|t-t_{k}\right|^{-\alpha_{k}},
$$

on $(-\pi, \pi)$ is true.

From this representation directly follows that if

$$
\alpha_{0} \in\left[\frac{1}{q}, 1+\frac{1}{q}\right)
$$

then the system (3) belongs to the space $L_{q}(-\pi, \pi)$. Then from the relation (4) we obtain that the system $\left\{E_{n}(\mu)\right\}_{n \neq 0}$ is minimal in $L_{q}(-\pi, \pi)$.

Consider the completeness of system

$$
\left\{\mu(t) \mathrm{e}^{\mathrm{int}}\right\}_{n \neq 0},
$$

in $L_{q}(-\pi, \pi)$. Let for some function $f \in L_{q}(\pi, \pi)$ we have

$$
\int_{-\pi}^{\pi} \mu(t) \mathrm{e}^{\mathrm{int}} \overline{f(t)} \mathrm{d} t=0, \forall n \neq 0 .
$$

Since $\mu f \in L_{1}(-\pi, \pi)$, then from this relation follows that

$$
\mu f \equiv \text { const } \Rightarrow f=\frac{c}{\mu},
$$

where $c$ is some constant. It is clear that

$$
\mu^{-1} \notin L_{q}(-\pi, \pi) \text {, }
$$

so $\alpha_{0}>\frac{1}{q}$. Consequently, $c=0$, hence $f=0$. As a result we obtain that under the following conditions

$$
\alpha_{0} \in\left[\frac{1}{q}, 1+\frac{1}{q}\right),\left\{\alpha_{k}\right\}_{1}^{r} \subset\left(-\frac{1}{p}, \frac{1}{q}\right),
$$

system (5) is complete and minimal in $L_{q}(-\pi, \pi)$. Thus, the system (1) is complete, but it is not minimal in $L_{q}(-\pi, \pi)$.

Consider the basicity of system (5) in $L_{q}(-\pi, \pi)$. If the conditions

$$
\left\{\alpha_{k}\right\}_{0}^{r} \subset\left(-\frac{1}{p}, \frac{1}{q}\right),
$$

satisfies, then it is known that (see. e.g. [9-13]) system (1) forms basis for $L_{q}(-\pi, \pi), 1<p<+\infty$, and in the case $p=1$ it is complete and minimal in $L_{1}(-\pi, \pi)$. Then it is clear that system (5) is minimal, but is not complete in $L_{q}(-\pi, \pi)$.

Now, let the condition (6) holds. It is easy to see that the system

$$
h_{n}(t)=-\frac{1}{2 \pi \mu(t)}\left(\mathrm{e}^{\mathrm{int}}-1\right), \forall n \neq 0,
$$

is biorthogonal to the system (5) in $L_{q}(-\pi, \pi)$. Let us show that in this case the system (5) does not form a basis for $L_{q}(-\pi, \pi)$. Let it forms a basis for $L_{q}(-\pi, \pi)$. At first consider the case $\alpha_{0} \in\left(\frac{1}{q}, 1+\frac{1}{q}\right)$. Then it is known that (see e.g. [16]) should fulfilled the following conditions

$$
0<\inf _{n \neq 0}\left\|E_{n}\right\|_{p}\left\|h_{n}\right\|_{q} \leq \sup _{n \neq 0}\left\|E_{n}\right\|_{p}\left\|h_{n}\right\|_{q}<+\infty,
$$

where \|\|$_{p}$ is an arbitrary norm for $L_{q}(-\pi, \pi)$. We have

$$
\left\|E_{n}\right\|_{p}=\left(\int_{-\pi}^{\pi} \mu^{p}(t) \mathrm{d} t\right)^{\frac{1}{p}}>0, \forall n \neq 0 .
$$

Regarding biorthogonal system we get the following condition

$$
0<\inf _{n \neq 0}\left\|h_{n}\right\|_{q} \leq \sup _{n \neq 0}\left\|h_{n}\right\|_{q}<+\infty .
$$

So

$$
\begin{aligned}
I_{n} & =\int_{-\pi}^{\pi}\left|h_{n}(t)\right|^{q} \mathrm{~d} t=\frac{1}{(2 \pi)^{q}} \int_{-\pi}^{\pi} \mu^{-q}(t)\left|\mathrm{e}^{\mathrm{int}}-1\right|^{q} \mathrm{~d} t \\
& =c_{p} \int_{-\pi}^{\pi} \frac{\left|\sin \frac{n t}{2}\right|^{\frac{q}{2}}}{\mu^{q}(t)} \mathrm{d} t,
\end{aligned}
$$

where $c_{p}$ is a constant depending only on $p$ (in sequel also). Choose $\delta>0$ as small as the interval $[0, \delta]$ does not contain the points $\left\{\alpha_{k}\right\}_{1}^{r}$. Then it is absolutely clear that $\exists m_{p}>0$ :

$$
\prod_{k=1}^{r}\left|t-t_{k}\right|^{-\alpha_{k} q} \geq m_{p}, \forall t \in[0, \delta] .
$$


Consequently

$$
\begin{aligned}
I_{n} & \geq c_{p} \int_{0}^{\delta} \frac{\left|\sin \frac{n t}{2}\right|^{\frac{q}{2}}}{\mu^{q}(t)} \mathrm{d} t \geq c_{p} m_{p} \int_{0}^{\delta} \frac{\left|\sin \frac{n t}{2}\right|^{\frac{q}{2}}}{t^{\alpha_{0} q}} \mathrm{~d} t \\
& =c_{p} m_{p} \int_{0}^{\frac{n}{2} \delta} \frac{|\sin \tau|^{\frac{q}{2}}}{\left(\frac{2}{n}\right)^{\alpha_{0} q} \tau^{\alpha_{0} q}} \frac{2}{n} \mathrm{~d} \tau \\
& =c_{p} m_{p} n^{\alpha_{0} q-1} \int_{0}^{\frac{n}{2} \delta} \frac{|\sin \tau|^{\frac{q}{2}}}{\tau^{\alpha_{0} q}} \mathrm{~d} \tau .
\end{aligned}
$$

It is clear that for sufficiently great $n$ we have $\frac{n \delta}{2}>1$. Thus

$$
I_{n} \geq c_{p} m_{p} n^{\alpha_{0} q-1} \int_{0}^{1} \frac{|\sin \tau|^{\frac{q}{2}}}{\tau^{\alpha_{0} q}} \mathrm{~d} \tau \rightarrow \infty, n \rightarrow \infty,
$$

so $\alpha_{0} q-1>0$. And it contradict the condition (7).

Consider the case $\alpha_{0}=\frac{1}{q}$. In the absolutely same way as in the previous case, we get

$$
I_{n} \geq c_{p} m_{p} \int_{0}^{\frac{n \delta}{2}} \frac{|\sin \tau|^{\frac{q}{2}}}{\tau} \mathrm{d} \tau .
$$

Hence it directly follows that

$$
\sup _{n} I_{n} \geq c_{p} m_{p} \int_{0}^{+\infty} \frac{|\sin \tau|^{\frac{q}{2}}}{\tau} \mathrm{d} \tau \geq c_{p} m_{p} \int_{1}^{+\infty} \frac{|\sin \tau|^{\frac{q}{2}}}{\tau} \mathrm{d} \tau .
$$

Consider the case $1<q \leq 2$. We have

$$
\sup _{n} I_{n} \geq c_{p} m_{p} \int_{1}^{+\infty} \frac{|\sin \tau|}{\tau} \mathrm{d} \tau=+\infty .
$$

Let $q>2$. Take $m \in N: q \leq 2^{m}$. Consequently

$$
\sup _{n} I_{n} \geq c_{p} m_{p} \int_{1}^{+\infty} \frac{|\sin \tau|^{\frac{q}{2}}}{\tau} \mathrm{d} \tau \geq c_{p} m_{p} \int_{1}^{+\infty} \frac{|\sin \tau|^{2^{m}}}{\tau} \mathrm{d} \tau .
$$

In the sequel we should pay attention to the following identities

$$
\begin{aligned}
\sin ^{2} \tau & =\frac{1}{2}(1-\cos 2 \tau),(1-\cos 2 \tau)^{2} \\
& =\frac{3}{2}-2 \cos 2 \tau+\frac{1}{2} \cos 4 \tau .
\end{aligned}
$$

From these relations and from the fact that the product of cosines expressed in terms of cosines, it directly follows

$$
\sin ^{2^{m}} \tau=a_{0}+a_{1} \cos b_{1} \tau+\cdots+a_{2^{m}} \cos b_{2^{m}} \tau,
$$

where $a_{k} ; \quad b_{k} k=\overline{1,2^{m}}$, are some constants. It is easy to see that $a_{0}, b_{k}>0$. Taking into account the expressions above we have

$$
\sup _{n} I_{n} \geq c_{p} m_{p}\left[\int_{1}^{+\infty} \frac{a_{0} \mathrm{~d} \tau}{\tau}+\sum_{k=1}^{2^{m}} c_{k} \int_{1}^{+\infty} \frac{\cos b_{k} \tau}{\tau} \mathrm{d} \tau\right] .
$$

It is clear that integrals $\int_{1}^{+\infty} \frac{\cos b_{k} \tau}{\tau} \mathrm{d} \tau$ converge. Then from the previous inequality follows that $\sup I_{n}=+\infty$. Thus, the following theorem is true.

Theorem 1. Let the following condition be satisfied

$$
\left\{\alpha_{k}\right\}_{k=0}^{r} \subset\left(-\frac{1}{p}, \frac{1}{q}\right) .
$$

Then the system $\left\{E_{n}(\mu)\right\}_{n \in \mathbf{Z}}$ forms a basis for $L_{q}(-\pi, \pi), 1<p<+\infty$. If the relation (6) holds, then this system is complete, but is not minimal in $L_{q}(-\pi, \pi)$, $1<p<+\infty$. In this case system (5) is complete and minimal in $L_{q}(-\pi, \pi)$ but does not form a basis for it.

The following theorem is also true.

Theorem 2. Let the conditions

$$
\left\{\alpha_{k}\right\}_{k=0}^{r} \subset(-1,0],
$$

be satisfied. Then the system $\left\{E_{n}(\mu)\right\}_{n \in \mathbb{Z}}$ is complete and minimal in $L_{1}(-\pi, \pi)$, but does not form a basis in it. If the conditions

$$
\alpha_{0} \in(0,1],\left\{\alpha_{k}\right\}_{1}^{r} \subset(-1,0],
$$

hold, then system (5) is complete and minimal in $L_{1}(-\pi, \pi)$, but does not form a basis for it.

Proof. If the conditions (8) holds then the system $\left\{E_{n}(\mu)\right\}_{n \in \mathbf{Z}}$ is complete and minimal in $L_{1}(-\pi, \pi)$. Indeed, it is clear that

$$
\exists p_{0} \in(1,+\infty):\left\{\alpha_{k}\right\}_{k=0}^{r} \subset\left(-\frac{1}{p_{0}}, \frac{1}{q_{0}}\right) .
$$

Consequently, the system $\left\{E_{n}(\mu)\right\}_{n \in \mathbf{Z}}$ forms a basis for $L_{p_{0}}(-\pi, \pi)$, and as a result it is complete in $L_{1}(-\pi, \pi)$. $\left\{E_{n}\left(\mu^{-1}\right)\right\}_{n \in \mathbf{Z}}$ is a biorthogonal system to $\left\{E_{n}(\mu)\right\}_{n \in \mathbf{Z}}$. It is clear that the system $\left\{E_{n}\left(\mu^{-1}\right)\right\}_{n \in \mathbf{Z}}$ belongs to $L_{\infty}(-\pi, \pi)$, and consequently, it is minimal in $L_{1}(-\pi, \pi)$. So the singular operator with the Hilbert kernel is not bounded in $L_{1}(-\pi, \pi)$, then as a result it follows that this system does not form a basis for $L_{1}(-\pi, \pi)$. If the conditions (9) hold then in the absolutely same way as in the previous case we establish that the system (5) is complete and minimal in $L_{1}(-\pi, \pi)$, but does not form a basis for it. Consequently, the system $\left\{E_{n}(\mu)\right\}_{n \in \mathbf{Z}}$ is complete, but is not minimal in $L_{1}(-\pi, \pi)$, and has a defect equal to 1 . 
The theorem is proved.

\section{Acknowledgements}

The authors are thankful to the referees for their valuable comments.

\section{REFERENCES}

[1] A. Zigmund, "Trigonometric Series," Vol. 1, Mir, Moscow, 1965.

[2] A. Zigmund, "Trigonometric Series," Vol. 2, Mir, Moscow, 1965.

[3] R. Edwards, "Fourier Series in a Modern Exposition," Vol. 1, Mir, Moscow, 1985.

[4] R. Edwards, "Fourier Series in a Modern Exposition," Vol. 2, Mir, Moscow, 1985.

[5] N. K. Bari, "Biorthogonal Systems and Bases in Hilbert Space," Mathematics, Vol. 148, No. 4, 1951, pp. 69-107.

[6] K. I. Babenko, "On Conjugated Functions," DAN SSSR, Vol. 62, No. 2, 1948, pp. 157-160.

[7] V. F. Gaposhkin, "One Generalization of M. Riesz Theorem on Conjugated Functions," Matematicheskii Sbornik, Vol. 46, No. 3, 1958, pp. 111-115.

[8] K. S. Kazaryan and P. I. Lizorkin, "Multipliers, Bases and Unconditional Bases in the Weighted Spaces B and SB," Proceedings of the Steklov Institute of Mathematics, Vol.
187, 1989, pp. 111-130.

[9] E. I. Moiseev, "On Basicity of Systems of Cosines and Sines in Weight Space," Differential Equations, Vol. 34, No. 1, 1998, pp. 40-44.

[10] E. I. Moiseev, "The Basicity in the Weight Space of a System of Eigen Functions of a Differential Operator," Differential Equations, Vol. 35, No. 2, 1999, pp. 200-205.

[11] B. T. Bilalov and S. G. Veliyev, "On Completeness of Exponent System with Complex Coefficients in Weight Spaces," Transactions of the NAS of Azerbaijan, Vol. 25, No. 7, 2005, pp. 9-14.

[12] B. T. Bilalov and S. G. Veliyev, "Bases of Eigen Functions of Two Discontinuous Differential Operators," Differential Equations, Vol. 42, No. 9, 2006, pp. 190-192.

[13] S. S. Pukhov and A. M. Sedletskii, "Bases of Exponents, Sines and Cosines in Weight Spaces on Finite Interval," Doklady Akademii nauk. Rossijskâa akademiâ nauk, Vol. 425, No. 4, 2009, pp. 452-455.

[14] J. Garnett, "Bounded Analytic Functions," Mir, Moscow, 1984.

[15] E. S. Golubeva, "The System of Weighted Exponentials with Power Weights," Natural Sciences, Vol. 83, No. 2, 2011, pp. 15-25.

[16] M. G. Krein, "Functional Analysis," Nauka, Moscow, 1972. 